\title{
Middleware Support for Mobile Social Ecosystems
}

\author{
Alessandra Toninelli, Animesh Pathak, Amir Seyedi, Roberto Speicys Cardoso, and Valérie Issarny \\ \{alessandra.toninelli, animesh.pathak, amir.seyedi, roberto.speicys_cardoso, valerie.issarny\}@inria.fr \\ INRIA Paris-Rocquencourt, France
}

\begin{abstract}
With the increased prevalence of advanced mobile devices (the so-called "smart" phones), interest has grown in mobile social ecosystems, where users not only access traditional Web-based social networks using their mobile devices, but are also able to use the context information provided by these devices to further enrich their interactions. Owing to the large variety of platforms available for smart phones, as well as the different ways that data and context information is represented, it is natural to think of middleware solutions that the developers of these systems can use while creating their applications.

In this paper, we highlight the issues which should be addressed by middleware designed for mobile social ecosystems, taking into account the heterogeneity of both deployment nodes and available data, the intrinsic distributed nature of mobile social applications, as well as users' security concerns. As part of our ongoing effort to develop this middleware, we present a comprehensive model to represent mobile social ecosystems and the interactions possible in them, and show how to exploit it in a representative scenario.
\end{abstract}

\section{INTRODUCTION}

Social ties such as friendship, common interests, and shared professional activities are central to humans as they bind individuals together. This web of social bindings is referred to as a social network. Recent technological advances in wireless networks and the increasing availability of portable devices offer a unique chance to improve social applications, i.e., applications that support human social interactions and are characterized by their swarming, transitory, and informal qualities [3], [7]. The formation of ad hoc networks enables social encounters between proximate users with common interests, anywhere and anytime [3], [15]. A salient feature of these situations is that physical places can also act as social filters: e.g., a conference venue groups together people who are likely to share common interests. Several recent prototypes exploit individuals' co-location and reciprocal proximity for guiding social network formation and management strategies and for restricting the scope of user interactions [2], [15], but there is still a need for models to adequately represent the complexities arising from interactions with content (commenting, tagging, etc.), as well as formation of groups and organization of events. We propose the term mobile social ecosystem (MSE) to describe this richer set of interactions occuring between the participants in these situations. Supporting MSE applications raises several challenges, which are the focus of this paper.

In order to better illustrate the potential of mobile social ecosystems, and the challenges associated with designing middleware for managing them, we present a detailed discussion

This work was funded in part by an ERCIM Alain Bensoussan Fellowship Programme and the EU FP7 ICT FET CONNECT project. of a representative scenario. Our example revolves around a fictitious professional event, the European Conference for Young Researchers (ECYR), being organized in Amsterdam, the Netherlands. The conference consists of keynote talks, technical sessions, and breakout sessions for birds-of-a-feather to discuss topics of common interest. We assume that a certain amount of information, both about the event as well as the participants, is known at the beginning of the conference. As the meeting progresses, the users can gather more data, and use it to augment their view of the social ecosystem. Specifically, the following information is known a priori by everyone:

- Topics of Interest. In order to better match attendees to sessions, the conference provides a list of topics of interest. These are 1) Functional Programming, 2) Sensor Networks, 3) Android Mobile Phone OS, 4) Ceramics, 5) Formal Methods, and 6) Biotechnology.

- Participant List. The organizers of the conference compile a participant list which includes a speaker list that contains public information about the conference speakers and authors, and a pointer to an online professional profile (we have chosen LinkedIn public IDs in this example); and the information provided by each (non-speaker) attendee. Each attendee, in addition to the speaker list, had access to a subset of the participant list, compiled by combining his existing social relations, new acquaintances made at the conference, or possibly an official list provided by the conference organizers. In the sample list shown in Table I, rows $1-4$ represent the speaker list while attendee \#6 may also be aware of the information of attendee \#5, due to their being colleagues.

- Conference Schedule. This contains the list of events planned during the day, and their details include the presenters, location, timing, and theme, as relevant. A sample schedule can be seen in Table II.

- Locations. The list of locations available for activities during the conference. This is used to annotate both publicly available information such as the schedule, as well as for people to annotate their personal data such as location of stay, which they can then share with other participants whom they trust.

During the course of the conference, we envisage that the users will use the above information to perform the following activities, among others:

- Look up information on the research profiles of speakers and fellow attendees, subject to their privacy settings;

- Use the system's recommendation (based on shared topics 
TABLE I

PARTICIPANT LIST AT ECYR

\begin{tabular}{|c|l|l|l|l|}
\hline S.No & Name & Affiliation & Interests & LinkedIn ID \\
\hline \hline 1 & Smith, John & Stanford U. & 1,2 & jsmith \\
\hline 2 & Xiao, Shen & CNR Pisa & $3,4,5$ & xiao \\
\hline 3 & Katz, Joseph & Fraunhofer & $1,4,6$ & jkatz \\
\hline 4 & Kulkarni, Arun & IIT Delhi & $3,4,6$ & arunk \\
\hline 5 & Toninelli, Alessandra & INRIA & $3,5,6$ & alessandra \\
\hline 6 & Pathak, Animesh & INRIA & 2,3 & animesh \\
\hline 7 & Sanders, Jessica & MIT & 4,6 & jessicas \\
\hline$\ldots$ & $\ldots$ & $\ldots$ & $\ldots$ & $\ldots$ \\
\hline
\end{tabular}

TABLE II

SCHEDULE OF ECYR

\begin{tabular}{|c|c|c|}
\hline Time & Event & Location \\
\hline 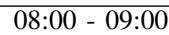 & Breakfast/Reception & Reception Hall \\
\hline $09: 00-10: 30$ & $\begin{array}{l}\text { Keynote: New Plings in Xab. } \\
\text { J.Smith, Stanford U. }\end{array}$ & Van Gogh Hall \\
\hline $10: 30-14: 00$ & Lunch & "The Wooden Shoe" \\
\hline $14: 00-15: 00$ & $\begin{array}{l}\text { Session I: "Computer Ceramics" } \\
\text { "Ceramics for iPhone", S. Xiao } \\
\text { and A. Conti (CNR Pisa) } \\
\text { "Cats on Pots: Android", A. } \\
\text { Kulkarni (IIT Delhi) and J. Katz } \\
\text { (Fraunhofer) }\end{array}$ & Room 5.A \\
\hline 15:00 - 16:00 & Coffee Break & Reception Hall \\
\hline $16: 00-17: 30$ & $\begin{array}{l}\text { Parallel Breakout Sessions } \\
\text { BS1: Topics } 1 \text { and } 5 \\
\text { BS2: Topics } 2 \text { and } 3\end{array}$ & $\begin{array}{l}\text { Room 5.A } \\
\text { Room 5.B }\end{array}$ \\
\hline
\end{tabular}

of interest) to meet up with fellow attendees;

- Make notes on the talks during the conference, and discuss with fellow attendees based on their comments;

- Plan shared taxi rides based on (newly shared) accommodation information;

- Plan spontaneous meetings based on common interests, and room availabilities; and

- Plan social events in Amsterdam in the evenings based on discovering interests of new acquaintences.

Complex mobile social ecosystems of the future, such as the one discussed in the scenario above, provide a rich platform for collaboration among individuals for achieving both professional and personal goals. In mobile social ecosystems, the heterogeneity of software platforms on constituent nodes, combined with their intrinsic distributed nature and heterogeneity of representation of data and context raises the need for middleware platforms to support the development of mobile social applications (MSAs).

Developing middleware for mobile social ecosystems is an extremely interesting and challenging research domain. Towards that end, in this paper, we make two contributions. Firstly, in Section II, we discuss in detail the various challenges in designing a middleware framework for MSE management. Following this, in Section III, we provide an expressive and extensible model of these social ecosystems, which can be used by middleware designers. We discuss related work on middleware for MSEs in Section IV, and conclude in Section V with a sketch of our planned research in the near future.

\section{Challenges for Middleware for Mobile Social ECOSYSTEMS}

The development of mobile social applications can be greatly simplified by the presence of middleware support. Middleware would be responsible for collecting, maintaining and processing social data, as well as allowing access to those data via flexible interfaces, thus enabling different applications to exploit information provided by the mobile social ecosystem. The design and development of a middleware support platform for mobile social applications (or a MSE management middleware), however, is challenging and must address a number of issues. In the following, we discuss the features which must be provided by middleware for these applications:

Expressive and flexible models to represent mobile social ecosystems: Existing social network models [4], [26] and social platforms [5] mostly define social interactions in terms of generic friendship relations between users, while not taking into account contextual information that might be very relevant on mobile platforms, such as proximity or location. An MSE management middleware should represent multiple aspects of users' social interactions by providing a comprehensive model that includes additional relations, such as content sharing and tagging, co-location, proximity, group membership, event participation and common interests.

Additionally, most existing Web-based social platforms, such as Facebook [5] or LinkedIn [20] are designed for a specific purpose and do not allow data sharing across different applications. When the need to enhance existing Web applications with social features arises, application developers often choose to define an ad-hoc representation for social information (e.g., YouTube [34]). This not only causes data redundancy, but also makes it very difficult to keep social data consistent across applications, while forcing developers to manage multiple representations of application-specific social information. The role of middleware is therefore to provide a shared model for social data representation, which allows different applications to produce, exchange and exploit social data with different purposes. This brings to the fore security and privacy issues, and the need to enforce access control policies, both between users and applications, as discussed next.

Privacy-awareness and access control: Mobile social applications manage contextual data, such as mobility traces, user preferences and activities, as well as human relationships, which are sensitive per se and can be further used to infer sensitive information. This raises critical security issues, particularly in terms of privacy and access control of users' data. Enforcing security in dynamic and open environments is always difficult as traditional models for distributed systems are not designed to face mobility issues. The task becomes even more difficult given the networked nature of MSAs, where information comes from multiple sources, moves to multiple destinations (possibly unforeseen at information production time) and is linked to other information following unpredictable patterns. We claim that security issues should 
be taken into account from the design phase of a MSE management middleware, affecting both the data model and the platform architecture. For instance, users should be enabled to store social information in their own devices and have full control over data disclosure. In addition, appropriate access control policies should be defined and enforced over social data exchange [30].

MSE management functionalities: MSE management includes functionalities for (a) MSE creation, and (b) MSE updates. Information describing each user's MSE may be gathered from existing social applications (e.g., a Facebook friendship link), but also by analyzing available contextual information, such as users' proximity patterns [19], [24] or mobile phone usage traces [4]. As human relations evolve, data describing MSE must change to represent this dynamics. In particular, the topology of social connections in mobile environments might vary not only because of changes in users' relations (e.g., users strengthening their ties or meeting new people), but also because of their mobility. In addition, since mobile devices are resource-constrained, algorithms for adding, removing or modifying data in a user's MSE must be efficient. For example, existing work on network topology (e.g., scale-free networks [1]) may provide useful background and inspiration to design novel algorithms for the dynamic removal of nodes from mobile social ecosystems.

Fully distributed architecture: Mobile environments are naturally distributed, and users must be able to access their MSE data anywhere and anytime. Centralized architectures used by current Web social applications and platforms, thus, are not appropriate for the mobile setting. Therefore, MSE management middleware should be designed in a fully distributed fashion to exploit mobile ad-hoc connectivity, whenever and wherever available, and without assuming centralized servers. The middleware should also provide protocols and algorithms that enable user applications to benefit from MSE information even if only provided with its partial view. A global view of users' MSE may not (always) be available, while privacy and portability issues might discourage approaches based on MSE data replication on each user's device. Therefore, novel algorithms and techniques are needed to allow users to add, remove and exchange relevant parts of their MSE data with other users.

In this section we motivated the need for novel research on MSE management middleware. In the following we show our initial effort in this direction by providing a representational model of social data for MSE management middleware.

\section{Modeling Mobile Social Ecosystems}

Despite their intuitive meaning in everyday life, social ecosystems might be very challenging to represent by means of a formal model. Graph theory, which offers a natural solution to model social networks, is in fact the most common approach to represent social relationships in the state of the art. The initial quantitative approaches to social networks, such as [16], were inspired by "small world" models, where the social network is essentially modeled as a random graph with adjusted degree distribution [23]. Relevant work has also been dedicated to analyzing the properties of social networks based on graph topology and dynamic evolution [22].

To the best of our knowledge, all existing work in this social models relies on some variation of a basic "social relation" model, where nodes are people (or more generally agents) and edges represent a generic link between them [18], [23]. More complex models take time into account by defining edges between nodes as state functions [33], or assign different weights to the edges of a social graph, in order to express a variable degree of tie strength [17]. However, they do not explicitly distinguish different types of social ties, from real friendship to acquaintance, from specific relationships (e.g., colleagues) to preference/interest sharing. Real social ecosystems, however, are more complex than represented in current models. This is because several activities and interactions of users actually have a social meaning, although they are not generally considered as being part of social networks. For example, user profiles adopted in several mobile context-aware applications often contain socially relevant information, such as interests and preferences, but also affiliation to organizations or groups. This seems to suggest that some of these categories should find their place in a proper MSE model.

Another interesting contribution is provided by research in the field of spontaneous ontology development by online users, i.e., folksonomy [21]. Folksonomies are taxonomies describing a specific domain of knowledge, which are built by connecting relevant concepts created by users and assigned to online content via tagging (e.g., on Flickr). By integrating the social dimension with the existing models for folksonomies, it is possible to link online users with the content they tagged, thus creating a tripartite graph of actors, concepts and instances [21]. However, since these models are conceived for online content and user modeling, they do not generally take into account users' dynamic characteristics, such as proximity and location, as well as the specific issues/potentials of MSAs (e.g., building location-based social ecosystems).

\section{A. A Comprehensive Model for Mobile Social Ecosystems}

In order to provide a suitable trade-off between formal MSE modeling and application-specific concepts, we define a set of basic entities acting as building blocks for the creation of mobile social ecosystems. We call these entities first-class entities, meaning that they are necessary and sufficient to describe any mobile social ecosystem, albeit in a general way. We derived these entities from the analysis of (i) real world use case scenarios, like the one described in Section I, and other scenarios presented by existing work on mobile social networks [2]; (ii) existing models for representing not only social networks, but also networks of contents, such as folksonomies [21]; and (iii) previous experience on knowledge representation, particularly with respect to semantic languages to represent social information [30]. The model defines five first-class entities, namely: 


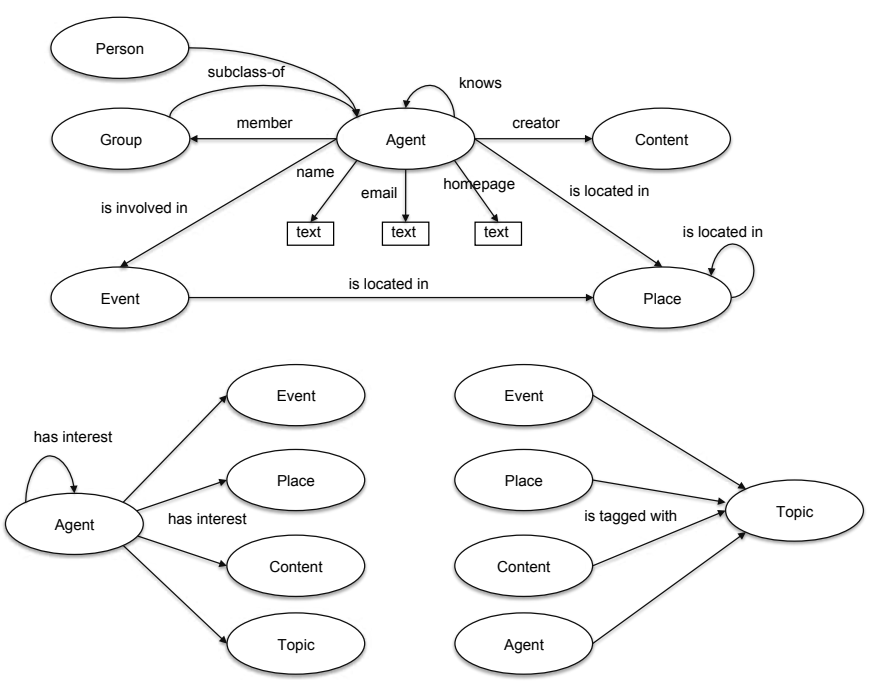

Fig. 1. First-class Entities and Relationships

Agent. An entity having beliefs, desires, intentions, according to the BDI design model for intelligent agents [29]. Subclasses of Agent are Person and Group. A Group is a set of Persons. Person and Group are used in practice more frequently than the generic Agent class. However, their superclass is needed to collect relevant properties and relationships that are common to both classes.

Event. In general, a 4D occurrence, i.e., an entity whose existence in characterized in tridimensional space and time [12]. Among others, social events, such as meetings, represent an interesting type of event. Events are not, however, bound to any specific social setting: for example, a person entering a room may be modeled as an event if this had a social meaning to the application. As events have a temporal dimension, they can be either atomic, or the temporal composition of two or more events (either in sequence or in parallel).

Place. A physical entity located in a space. This concept is especially needed to model mobile social ecosystems, where physical location plays an explicit role in social activities.

Content. An entity that conveys some kind of information. It could also be defined as an information object, such as a picture, a blog post or a speech.

Topic. A subject of interest for users in mobile social ecosystems. This concept definition was mainly driven by common application scenarios, where users "tag" contents online with keywords and categories (e.g., folksonomies).

Among the above defined classes several binary relations might hold, including self-referential properties. We define a subset of fundamental relations that we call first-class relationships. First-class relationships reflect an essential and invariant relation between the two considered entities (or the same entity in case of self-referential). For example, an agent's relation with an event is always some kind of involvement: depending on the particular situation, the agent might be the presenter of a talk or the organizer of a party. Other relations may be added to first-class ones, some of which being fundamental to describe specific applications. For instance, by defining Picture as a subclass of Content and defining the depicts relationship, one might state that a picture depicts a place. However, given the application-dependent nature of those relations, we do not include them in the first-class set.

Figure 1 shows the graph of first-class entities and relationships. The graph should be interpreted as the set of base relations that may hold between a set of first-class entities and it reads as follows. An agent may be involved in an event; may be located in a place; may know another agent; may be the creator of a content; and may belong to a group. A place may be located in another place. An event may also be located in a place. An agent may be interested in any event, place, topic and content. Agents, places, events and contents may be tagged with a topic. The model also includes a set of attributes that provide information about an agent, such as name, email and homepage. These attributes were inspired by the Friendof-a-Friend (FOAF) ontology, one of the very few existing standards for expressing socially relevant information [6].

We represent our MSE model by adopting the Resource Description Framework $(\mathrm{RDF})^{1}$, a base Semantic Web standard. The data model of RDF is very generic and is well suited to modeling real-world phenomena, including entities, events and interactions that characterize mobile social ecosystems. RDF enables simple reasoning over data, and thus supports the association of formal semantics with data models. At the same time, RDF encourages modeling using semi-structured data, alleviating the need for a strict, predefined schema. In particular, the MSE, as described above, is represented as a graph of RDF triples, i.e. subject-predicate-object triples, with each statement describing an attribute and its value. The set of RDF statements defining social information is linked as a graph of nodes and arcs, as shown in Figure 1. Agent's attributes have been modeled as RDF datatype properties and mapped onto FOAF corresponding properties, thanks to the common RDF vocabulary. This allows the direct import of existing FOAF profiles into our model ${ }^{2}$, with no additional effort. In case richer expressivity is needed (e.g., defining constraints on the cardinality or range of a property), ontologies can be easily augmented with Web Ontology Language (OWL) constructs. Given OWL's greater complexity, we limit our initial model to RDF, while keeping the possibility to upgrade to OWL whenever needed.

As a final remark, we note that our model provides firstclass entities and relationships to the MSE application developer who wishes to exploit them with no additional effort. The model does not, however, tightly constrain the usage of available classes and properties, nor does it prevent the developer from extending the base model to accomodate specific application requirements. This is mainly due to the flexibility of semantic languages, as shown in the following section. Additionally, because our model is based on a graph

\footnotetext{
${ }^{1}$ http://www.w3.org/TR/rdf-primer/

${ }^{2}$ Available at http://www-rocq.inria.fr/arles/mse/ontologies/mse.rdf
} 
representation, it is well suited to support the retrieval and representation of partial MSE knowledge since graphs can be split and merged based on users' application needs. Finally, existing quantitative models for social network analysis can be usefully applied to each (first-class) relationship, or possibly to a combination of then, to generate n-dimensional graphs.

\section{B. Applying the Model to a Real Use Case Scenario}

In this section we show how to concretely exploit our proposed model to describe mobile social ecosystems. In particular, by referring to the scenario introduced in Section I, we consider the following situation. Animesh is attending the second talk of Session I (see Table II), given by Joseph Katz. Arun Kulkarni is also attending his colleague's talk. Arun and Animesh introduce each other just before the talk and they exchange some basic information, including their LinkedIn profiles. By extracting keywords from the paper Arun is coauthor of, Animesh's application infers that they might share an interest for operating systems running on smart phones. Therefore, Animesh invites Arun to attend breakout session 2, and creates on-the-fly a discussion group for conference attendees interested in Android OS. Knowing that his colleague Alessandra is also interested in Android, Animesh invites her to join the group and proposes to group members to meet at the Reception Hall during the coffee break. Alessandra accepts both invitations, while Arun will not join the meeting because of a previous scheduled meeting.

Extending the base model: Let us consider Tables I and II. In order to translate data included in these tables into our model, a number of steps are required. The first step is extending the base model with application-specific classes and properties. Extension proceeds by subclassing RDF classes/properties and possibly importing other (portions of) ontologies, provided that they are compliant with the base model. In our example, we create subclasses of Event (Talk, Coffee Break and Meeting); subclasses of Group (Affiliation); and subclasses of Content (Paper and Keynote). We also create the corresponding property linking this new type of content to a person (subclass of agent), i.e., a person may be the speaker of a talk, and a person may attend a talk or meeting. A talk may present a paper or a keynote. We also create a subproperty of creator called admin, to express that a person may be the administrator of a group. We create a subproperty of homepage datatype property, called LinkedIn, referring to the personal homepage identifier on LinkedIn. Finally, we subclass the generic Place with more specific Room.

Creating entity instances: Now we can create instances of available classes. Based on Table I, we create instances of Person, e.g., "Arun Kulkarni" and "Animesh Pathak" each with its associated attributes (name and LinkedIn homepage identifier). We also created instances of Paper (e.g., "Cats on Pots: Android"), as well as the corresponding instances of Talk; of Places (e.g., "Room 5.A"); of Groups/Group subclasses (e.g, "INRIA" and "Android OS"); and a set of topics of interests for the conference attendees (e.g., "Android").

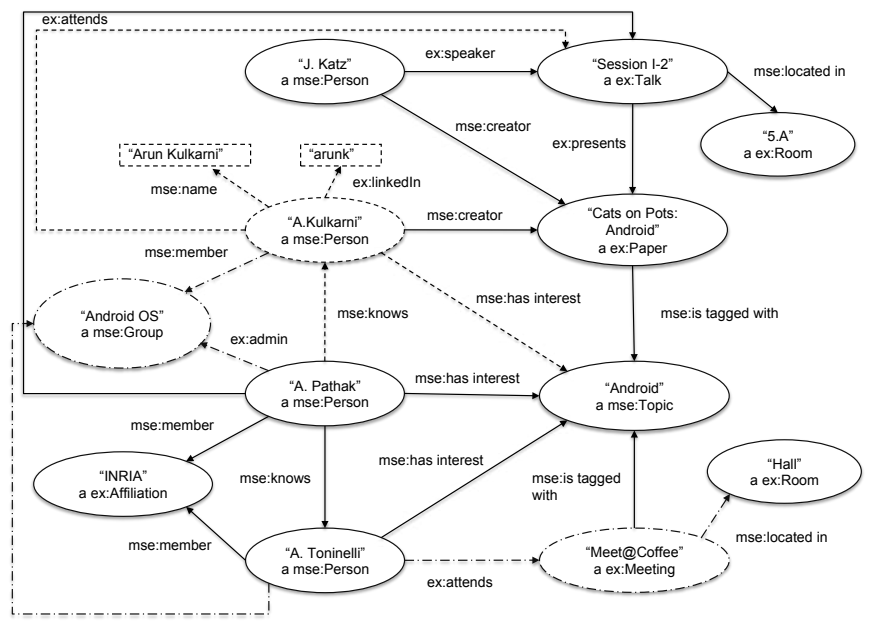

Fig. 2. Application of our Model to the ECYR Scenario

Connecting entities via social relationships: Finally, we link instances via appropriate properties according to the underlying ontological model. The resulting social graph is depicted in Figure 2. We adopt an N3-like notation ${ }^{3}$, where each individual's name is followed by the class it is an instance of, and define namespaces for the mobile social ecosystem model (mse: ) and the example extension (ex:). Note that the graph represents all social information known by Animesh: in particular, plain arrows/ovals are data known at the beginning, dashed arrows/ovals are portions of social graph acquired from Arun, and long dashed-dotted arrows/ovals are graph portions created by him during the interaction with Arun. Name and LinkedIn identifiers for Animesh and Alessandra are not shown in figure for the sake of clarity.

\section{RELATED WORK}

Web-based social networking applications have been rapidly spreading during the last few years, as shown by the success of Web-based social platforms, such as Facebook [5] and Twitter [32], as well as by the development of applications exploiting social information for various purposes, for example recommender systems based on social trust [8], [9]. Traditional Web applications, e.g., maps and email clients, have also been enhanced with social features [10], while recent efforts attempt to provide a uniform interface to access social information [25].

Moving from Web-based social communities to physical spaces, social applications specifically targeted at mobile environments have been developed in recent years. Current MSAs, such as applications supporting the dissemination of content updates (e.g., news or traffic information) over a mobile social network [14], or exploiting mobile social networking to enhance group communication [11], are often designed from scratch by embedding into the application logic all MSE management functionalities and providing application-specific

\footnotetext{
${ }^{3}$ http://www.w3.org/DesignIssues/Notation3
} 
data representation models. Some authors have recognized the need to externalize social management functionalities [28], [31], but to the best of our knowledge, only a few middleware frameworks to support MSAs have been proposed. The MobiSoc middleware supports the development of MSAs by allowing the sharing of social data between applications, as well as inferring new social knowledge from the observation of geo-social data [13]. MobiSoc centralized architecture, where a trusted server collects and manages all social information, is not suitable for modern MSEs, as discussed in Section II. In addition, MobiSoc only models profiles of people and places, while our model is able to represent more complex social interactions, including contents and events. Similarly, the MobiClique middleware, which supports social interaction between proximate users on mobile ad-hoc networks, only provides a very simple data model, focused on a specific application domain, and does not provide security features [27]. On the other hand, it represents an interesting effort to build a decentralized middleware architecture, which also provides social APIs. The semantic data model presented in [2], where the FOAF ontology is extended to model users' interests and activities, is the most similar to our RDF model. We take, however, a more comprehensive approach since our base ontology can integrate with FOAF, but also includes additional concepts. Moreover, their middleware is mainly focused on a specific application domain, that is, supporting mobile users in the accomplishments of their tasks, while our model is generic and well suited to represent various application domains.

Finally, several techniques and algorithms have been developed to extract meaningful social information from available context/location data, such as users' proximity patterns [19], [24] or mobile phone usage traces [4]. These techniques may be usefully integrated within a MSE management middleware to provide social information extraction and inference.

\section{CONCLUSIONS AND Future WORK}

In this paper, we discussed the challenges in designing middleware support for complex mobile social ecosystems of the not-so-distant future. We also presented the first results of our ongoing effort to develop this middleware, that is, an expressive and extensible model to represent MSEs and the interactions possible in them, and demonstrated its application in a representative scenario. Based on the proposed model, we are currently working on the design of a middleware framework to support mobile social ecosystems, and on the implementation of a prototype mobile social application that utilizes our middleware.

\section{REFERENCES}

[1] R. Albert, H. Jeong, and A.-L. Barabási. The diameter of the world wide web. CoRR, cond-mat/9907038, 1999.

[2] S. Ben Mokhtar and L. Capra. From pervasive to social computing: algorithms and deployments. In ICPS '09. ACM, 2009.

[3] E. F. Churchill and C. A. Halverson. Guest editors' introduction: Social networks and social networking. IEEE Internet Comput., 2005.

[4] N. Eagle, A. S. Pentland, and D. Lazer. Inferring friendship network structure by using mobile phone data. National Academy of Sciences, 106(36):15274-15278, Sept. 2009.
[5] Facebook, last visited: Mar 2010. http://www.facebook.com/.

[6] Friend of a Friend, last visited: Mar 2010. http://www.foaf-project.org/.

[7] M. Foth. Facilitating social networking in inner-city neighborhoods. IEEE Computer, 39(9):44-50, 2006.

[8] W. Geyer, C. Dugan, D. R. Millen, M. Muller, and J. Freyne. Recommending topics for self-descriptions in online user profiles. In RecSys '08, pages 59-66, New York, NY, USA, 2008. ACM.

[9] J. Golbeck and J. Hendler. FilmTrust: movie recommendations using trust in web-based social networks. In CCNC 2006. 3rd IEEE, 2006

[10] Google buzz, last visited: Mar 2010. http://www.google.com/buzz.

[11] R. Grob, M. Kuhn, R. Wattenhofer, and M. Wirz. Cluestr: mobile social networking for enhanced group communication. In GROUP 'O9, pp. 81-90, New York, NY, USA, 2009. ACM.

[12] N. Guarino and C. A. Welty. An overview of ontoclean. In S. Staab and R. Studer, editors, Handbook on Ontologies, International Handbooks on Information Systems, pp. 151-172. Springer, 2004.

[13] A. Gupta, A. Kalra, D. Boston, and C. Borcea. MobiSoC: a middleware for mobile social computing applications. Mob. Netw. Appl., 2009.

[14] S. Ioannidis, A. Chaintreau, and L. Massoulié. Distributing content updates over a mobile social network. ACM SIGMOBILE MC2R, 13(1):44-47, 2009.

[15] Q. Jones and S. A. Grandhi. P3 systems: Putting the place back into social networks. IEEE Internet Comput., 9(5):38-46, 2005.

[16] J. Kleinberg. The small-world phenomenon: An algorithmic perspective. In 32nd ACM Symposium on Theory of Computing, 2000.

[17] J. M. Kumpula, J. P. Onnela, J. Saramäki, K. Kaski, and J. Kertész. Emergence of communities in weighted networks. Physical Review Letters, 99(22), 2007.

[18] J. Leskovec, K. J. Lang, A. Dasgupta, and M. W. Mahoney. Statistical properties of community structure in large social and information networks. In $W W W$ '08, pp. 695-704, New York, NY, USA, 2008. ACM.

[19] N. Li and G. Chen. Multi-layered friendship modeling for locationbased mobile social networks. In MobiQuitous '09, pages 1-10, July 2009.

[20] Linked In, last visited: Mar 2010. http://linkedin.com/.

[21] P. Mika. Ontologies are us: A unified model of social networks and semantics. Web Semant., 5(1):5-15, Mar 2007.

[22] A. Mislove, M. Marcon, K. P. Gummadi, P. Druschel, and B. Bhattacharjee. Measurement and analysis of online social networks. In $A C M$ SIGCOMM IMC '07, pages 29-42, New York, NY, USA, 2007. ACM.

[23] M. E. J. Newman, D. J. Watts, and S. H. Strogatz. Random graph models of social networks. In National Academy of Science, USA, volume 99, pages 2566-2572, 2002.

[24] A. Noulas, M. Musolesi, M. Pontil, and C. Mascolo. Inferring interests from mobility and social interactions. In NIPS Workshop on Analyzing Networks and Learning with Graphs, 2009.

[25] Open Social. http://code.google.com/apis/opensocial/.

[26] A. S. Pentland. Automatic mapping and modeling of human networks. Physica A: Statistical Mechanics and its Applications, May 2007.

[27] A.-K. Pietiläinen, E. Oliver, J. LeBrun, G. Varghese, J. Crowcroft, and C. Diot. Experiments in mobile social networking. TRCR-PRL-200802-0003, Thomson, Feb 2008.

[28] J. Rana, J. Kristiansson, J. Hallberg, and K. Synnes. An architecture for mobile social networking applications. In CICSYN ’09, July 2009.

[29] A. S. Rao and M. P. Georgeff. Modeling rational agents within a BDIarchitecture. In J. Allen, R. Fikes, and E. Sandewall, editors, KR 1991, pp. 473-484, 1991.

[30] A. Toninelli, R. Montanari, O. Lassila, and D. Khushraj. What's on users' minds? toward a usable smart phone security model. Pervasive Comput., IEEE, 8(2):32-39, Apr-Jun 2009.

[31] M. Tran, J. Han, and A. Colman. Social context: Supporting interaction awareness in ubiquitous environments. In MobiQuitous '09, pp. 1-10, Jul 2009.

[32] Twitter, last visited: Mar 2010. http://www.twitter.com.

[33] G. G. Van De Bunt, M. A. J. Van Duijn, and T. A. B. Snijders. Friendship networks through time: An actor-oriented dynamic statistical network model. Comput. Math. Organ. Theory, 5(2):167-192, 1999.

[34] You Tube, last visited: Mar 2010. http://youtube.com/. 